\title{
Three-Dimensional Visualization of the Coronary Venous System Using Multidetector Row Computed Tomography
}

\author{
Hiroshi Tada, MD; Kenji Kurosaki, MD; Shigeto Naito, MD; Keiko Koyama, MD*; \\ Kazuhiro Itoi, RT*; Sachiko Ito, MD; Marehiko Ueda, MD; \\ Goro Shinbo, MD; Hiroshi Hoshizaki, MD; Akihiko Nogami, MD**; \\ Shigeru Oshima, MD; Koichi Taniguchi, MD
}

\begin{abstract}
Background This study was undertaken to investigate the applicability and image quality of contrast-enhanced visualization of the coronary venous system (CVS) by multidetector row computed tomography (MDCT). Methods and Results A total of 70 patients underwent MDCT and for each patient, 6 data sets were created throughout the cardiac cycle. The number and location of coronary veins were evaluated in 3-dimensional images using the 6 data sets. The quality of all images reconstructed from the 6 data sets was too poor to evaluate the CVS in 6 patients (9\%). In the remaining 64 patients (91\%), the diameter of the CVS was usually greater in the images reconstructed from data acquired during systole than in those reconstructed from data acquired during diastole. However, artifacts were observed more often in images from systole than from diastole. The coronary sinus and middle cardiac vein were visible in all 64 patients. The left marginal and posterior veins also were identified in $54(84 \%)$ and 60 patients (94\%), respectively.
\end{abstract}

Conclusions MDCT can be used as a non-invasive modality for evaluating the CVS anatomy in most patients. (Circ J 2005; 69: 165-170)

Key Words: Computed tomography; Coronary vein; Three-dimensional images

$\mathbf{T}$ he coronary venous system (CVS) is increasingly being used for different electrophysiologic purposes; for example, cardiac resynchronization therapy incorporating a left ventricular (LV) coronary vein lead may improve the condition of patients with severe heart failure!-3 The CVS has also been used for radiofrequency catheter ablation, ${ }^{4}$ mapping5, and defibrillation, 6.7

Visualization of the CVS has been attempted by angiography, ${ }^{8,9}$ echocardiography ${ }^{10,11}$ and electron-beam computed tomography $(\mathrm{CT}), 12,13$ and recently contrast-enhanced multidetector row spiral computed tomography (MDCT) has been used to noninvasively visualize coronary artery stenosis by creating high-quality 3-dimensional (3-D) images ${ }^{14-19}$ However, there has not been a study to date that has attempted to analyze the CVS using MDCT, so the aim of the present study was to investigate the applicability and image quality of contrast-enhanced visualization of the CVS by MDCT using retrospective ECG gating. Attention was also focused on the presence and number of coronary veins on the lateral aspect of the left ventricle (LV), which are the optimal sites for placing pacing leads for LV pacing in patients with advanced heart failure.

(Received August 12, 2004; revised manuscript received November 17, 2004; accepted November 25, 2004)

Divisions of Cardiology, *Radiology, Gunma Prefectural Cardiovascular Center, Maebashi and **Division of Cardiology, Yokohama Rosai Hospital, Yokohama, Japan

Mailing address: Hiroshi Tada, MD, Division of Cardiology, Gunma Prefectural Cardiovascular Center, 3-12 Kameizumi, Maebashi, Gunma 371-0004, Japan. E-mail: tada.h@cvc.pref.gunma.jp

\section{Methods}

\section{Study Population}

This study included 70 patients who underwent MDCT as well as radiofrequency catheter ablation (51 men, 19 women; mean age, 58 \pm 9 years [range: $25-74$ years]). Sixtyfive patients had paroxysmal atrial fibrillation, and 5 had left atrial tachycardia. All patients underwent MDCT to determine the morphology and size of the left atrium and pulmonary veins prior to the ablation procedure 20 All patients had a normal ECG during sinus rhythm, and no structural abnormalities were found by physical examination and echocardiography.

\section{MDCT}

After informed written consent was obtained, MDCT was performed using a LightSpeed Ultra ${ }^{\mathrm{TM}}$ (GE Medical System, Milwaukee, WI, USA). All patients were in normal sinus rhythm at the time of MDCT image acquisition. Patients were examined while supine and all images were acquired during an inspiratory breath-hold. After determining the contrast agent transit time, we acquired MDCT data during an intravenous injection of $100 \mathrm{ml}$ of the iodinated contrast agent iopromid (Ultravist 370; Schering, Berlin, Germany) at a rate of $4 \mathrm{ml} / \mathrm{s}$. The following scanning protocol was used: 8-detector; beam collimation, $1.25 \mathrm{~mm}$; pitch factor, $0.275-0.35$; scan time, 0.5 or 0.6 s/rotation; ECG gate, and half- or multi-sector reconstruction ${ }^{17}$ The tube current was $150 \mathrm{~mA}$ at $140 \mathrm{kV}$ to keep the radiation dose within a reasonable range. On the basis of the results of the preliminary study, the data acquisition was started $25 \mathrm{~s}$ after the initiation of the contrast agent. The breath-hold 

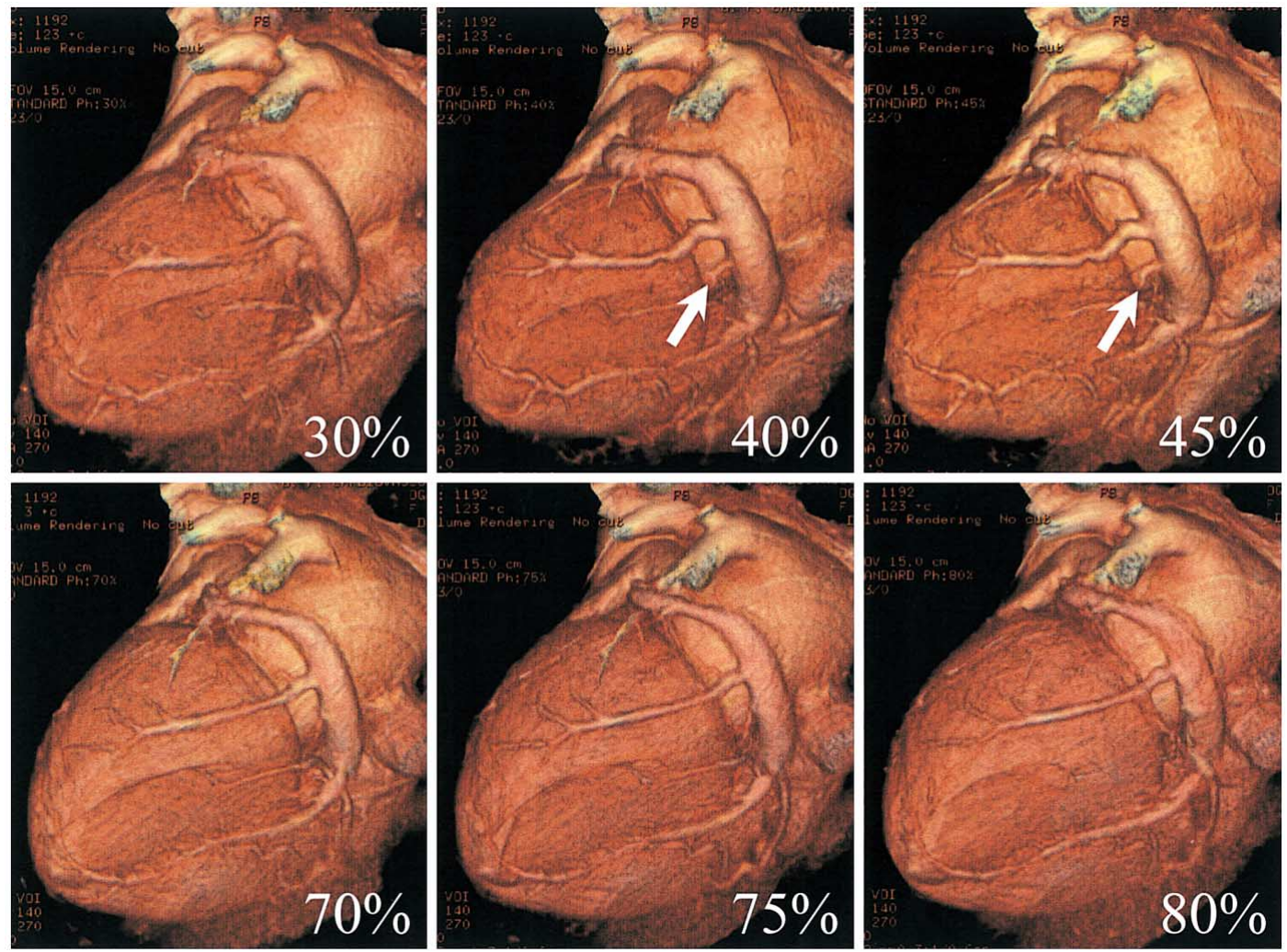

Fig 1. Representative reconstructed 3-dimensional (D) images of the coronary venous system throughout the cardiac cycle $(30 \%, 40 \%, 45 \%, 70 \%, 75 \%$, and $80 \%$ of the $\mathrm{R}$ wave-to-R wave interval). Note that the diameters of the coronary veins are greater in images reconstructed during systole than during diastole, and that a small branch originating from the coronary sinus (arrows) can be clearly detected only in the images reconstructed at $40 \%$ and $45 \%$ of the R wave-to-R wave interval.

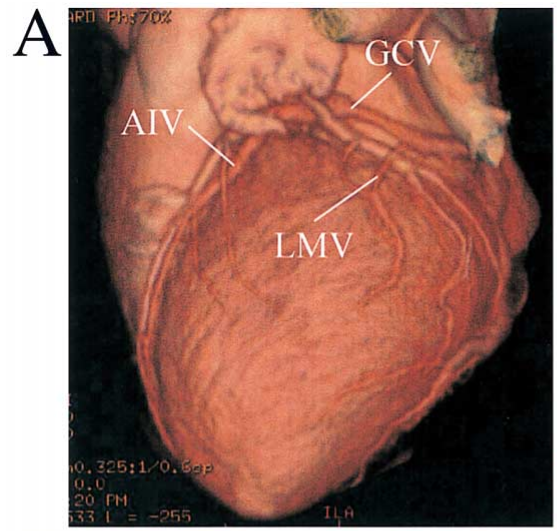

B

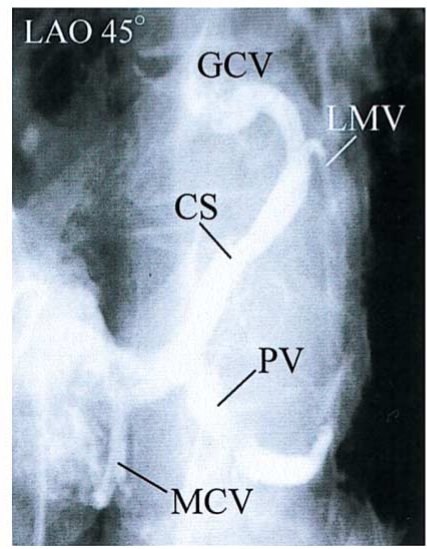

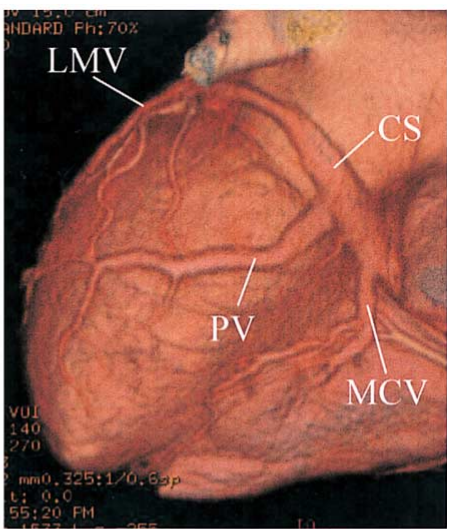

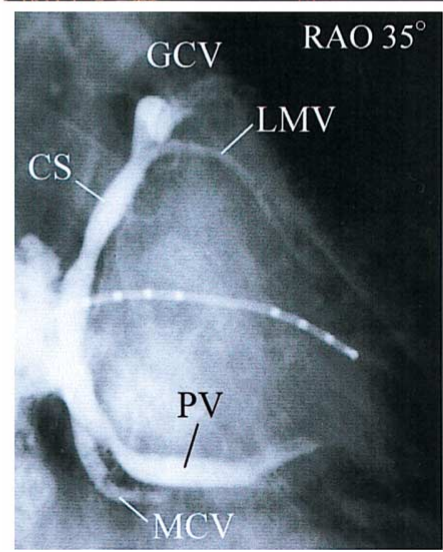

Fig 2. Representative reconstructed 3-D images (A) and venograms (B). CT images were reconstructed at $70 \%$ of the $\mathrm{R}$ wave-to-R wave interval. AIV, anterior interventricular vein; $\mathrm{CS}$, coronary sinus; $\mathrm{GCV}$, great cardiac vein; L(R)AO, left (right) anterior oblique projection; LMV, left marginal vein; MCV, middle cardiac vein; PV, posterior vein. 

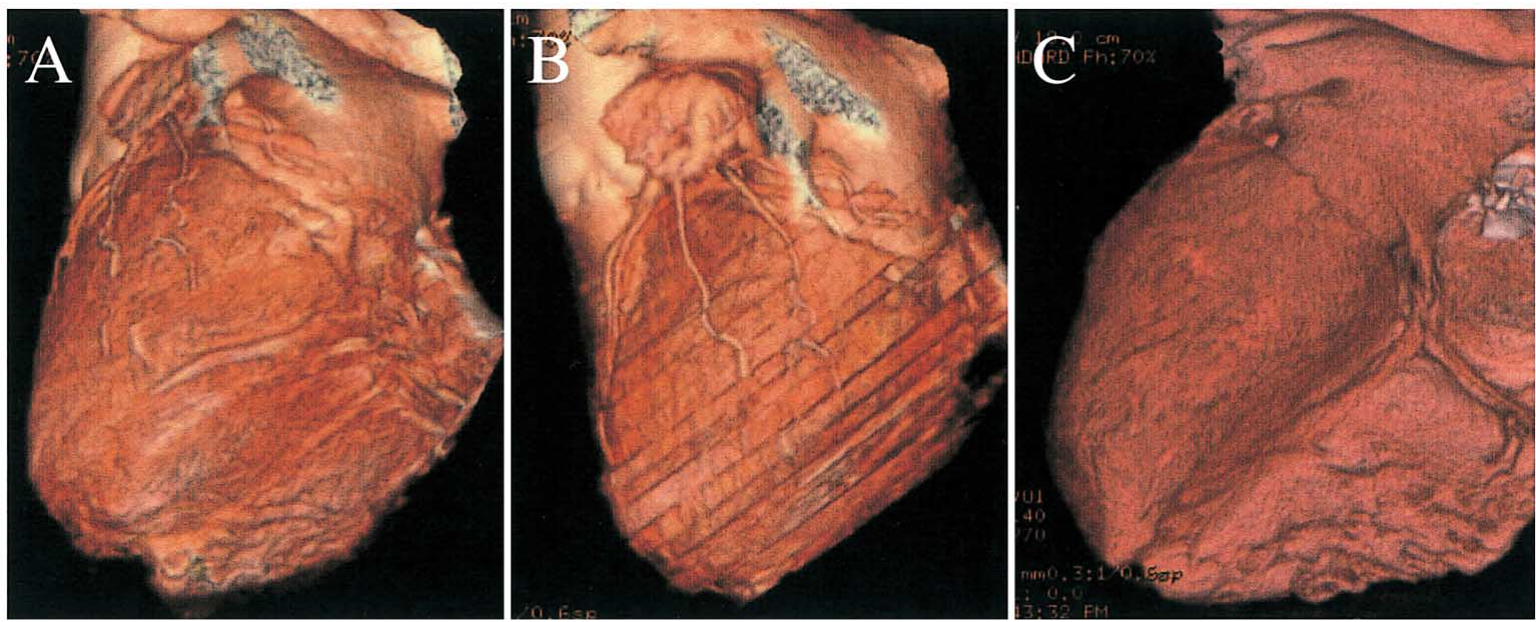

Fig 3. Example of images that are inappropriate for evaluating the coronary venous system. (A) Motion artifact because of arrhythmia (supraventricular contractions) during data acquisition. (B) Banding artifact. (C) Insufficient enhancement of the coronary venous system because of a small amount of contrast agent.
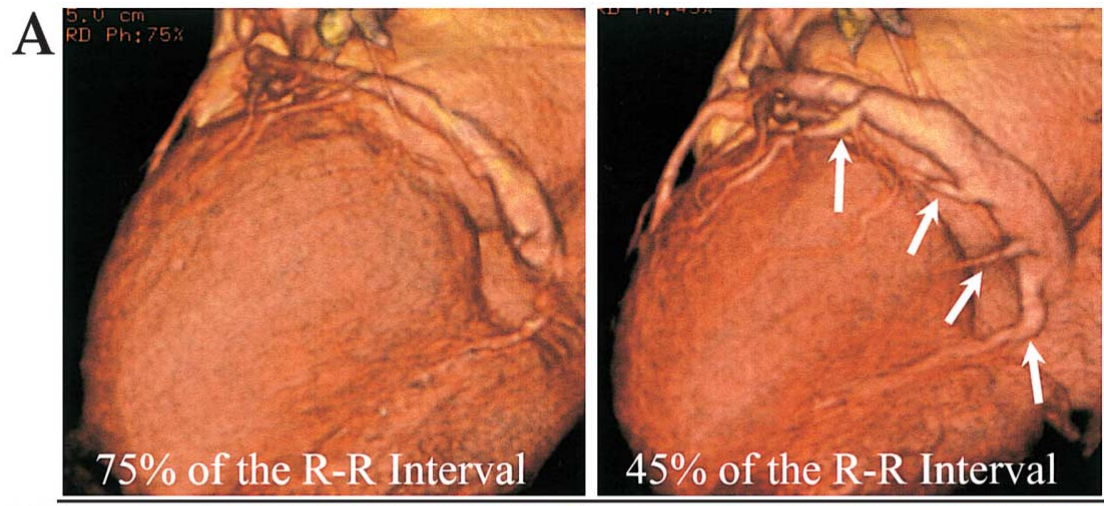

B
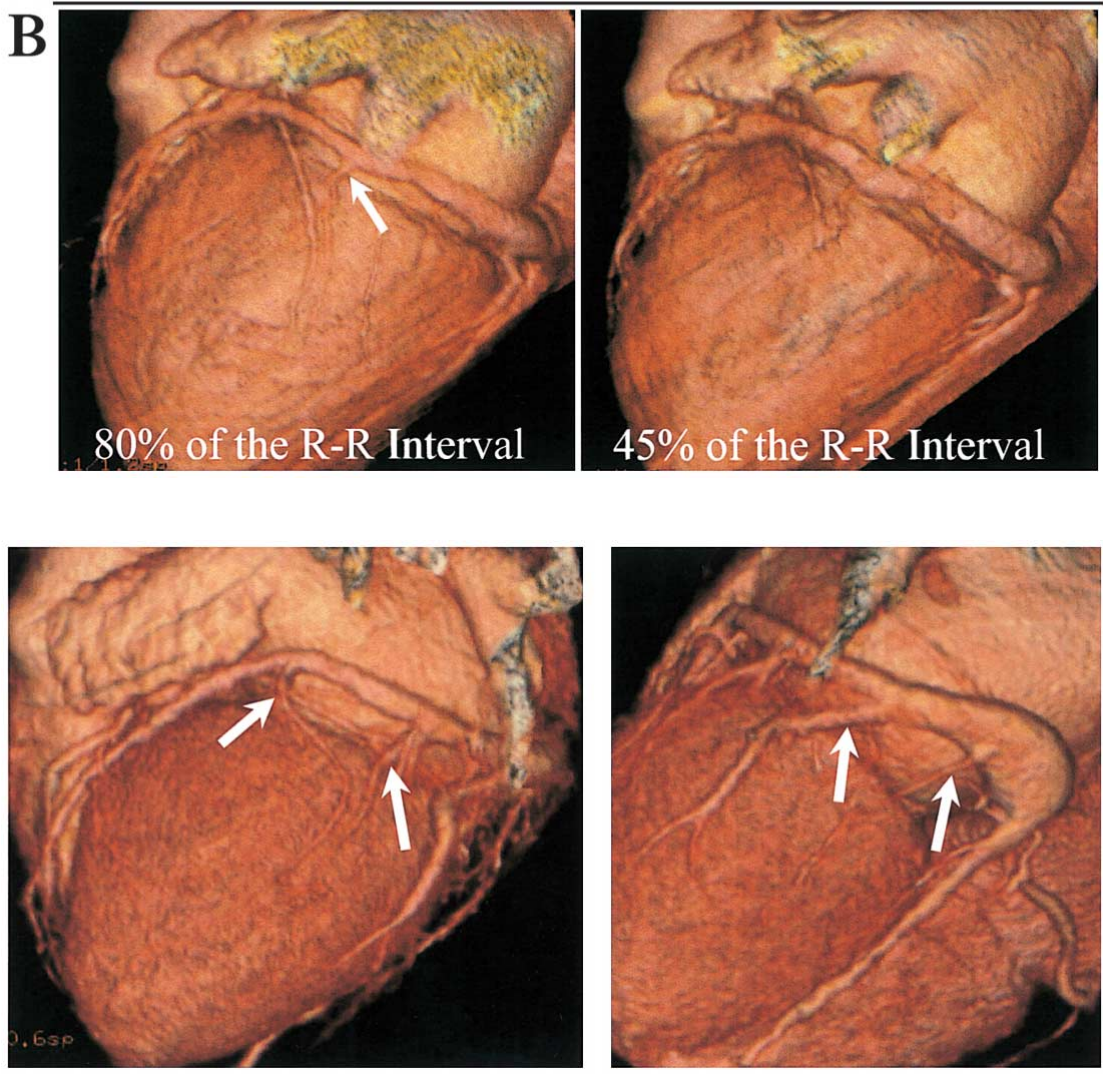

Fig 4. (A) A 3-D image demonstrating that the systolic data (Sys-Image: Right) is more suitable than the diastlic data (Dia-Image: Left) for evaluating the coronary venous system. A left marginal vein, 2 small posterior veins, and a middle cardiac vein are seen clearly on the image reconstructed at $45 \%$ of the $\mathrm{R}$ wave-to- $\mathrm{R}$ wave interval. (B) A 3-D image demonstrating that the DiaImage (Right) is more suitable than the SysImage (Left) for evaluating the coronary venous system. A left marginal vein and its course are seen clearly on the image reconstructed at $80 \%$ of the $\mathrm{R}$ wave-to- $\mathrm{R}$ wave interval.

Fig 5. Representative images reconstructed at $45 \%$ of the $R$ wave-to- $R$ wave interval demonstrating 4 cardiac veins (arrows) on the lateral aspect of the left ventricle. 
time was between 30 and $40 \mathrm{~s}$, depending on the scanning volume.

For 3-D image reconstruction, we processed the raw CT data on a separate workstation (Advanced Workstation 4.0; GE Medical System). Using ECG-gating and a $180^{\circ}$ multislice cardiac interpolation algorithm, we reconstructed cross-sectional images with a slice thickness of $1.2-1.4 \mathrm{~mm}$ in 1-mm intervals to obtain image acquisition windows of $125-250 \mathrm{~ms}$ (full width at one-tenth maximum of the phase-contribution profile), depending on the patient's heart rate. Half- or multi-sector reconstruction was used as a reconstruction algorithm. ${ }^{21}$ The field of view was $180 \mathrm{~mm}$ with an image matrix of $512 \times 512$ pixels. For each patient, 6 data sets were created throughout the cardiac cycle $(30 \%$, $40 \%, 45 \%, 70 \%, 75 \%$, and $80 \%$ of the $\mathrm{R}$ wave-to-R wave interval; Fig 1).

\section{Terminology}

The terminology used to describe the various tributary veins is based on previous studies (Fig 2) , $^{9}$

Anterior Interventricular Vein In most patients, the anterior interventricular vein (AIV) originates in the lower or middle third of the anterior interventricular groove. It follows the groove toward the base of the heart and then turns posteriorly at the atrioventricular groove to enter the great cardiac vein (GCV).

Middle Cardiac Vein The middle cardiac vein (MCV) originates close to the apex of the heart and follows the posterior interventricular groove toward the base. Usually, the MCV drains into the coronary sinus (CS) close to its ostium in the right atrium.

Posterior/Left Marginal Veins The posterior veins (PVs)/left marginal veins (LMVs) originate either from the posterior or lateral aspects of the $\mathrm{LV}$ and drain into the GCV or into the CS.

\section{Analysis of the CVS}

All 6 data sets were carefully examined to assess the presence and branching point of the tributary veins from the CS or GCV. The total number of PVs and LMV present on the antero-lateral or lateral wall of the LV also was assessed.

\section{Results}

\section{Image Quality}

In 64 of 70 patients (91\%), evaluation of the CVS was possible using the 6 reconstructed images, but not in the remaining 6 patients because the image quality was too poor as a result of motion artifact from arrhythmia during data acquisition or banding artifact (3 patients; Fig 3A,B) or insufficient enhancement of the CVS with little or no contrast agent (3 patients; Fig 3C).

\section{Influence of the Reconstruction Window on 3-D Images}

Because the diameters of the coronary veins were usually greater in images acquired during systole $(30 \%$, $40 \%$, and $45 \%$ of the $\mathrm{R}$ wave-to-R wave interval; SysImages) than during diastole $(70 \%, 75 \%$, and $80 \%$ of the $\mathrm{R}$ wave-to-R wave interval; Dia-Images), the Sys-Images were preferable for identifying the origin and course of the tributary veins from the CS. However, banding artifacts were observed more often in the Sys-Images than in the Dia-Images, and the presence of the LMV could not be judged from the Sys-Images in $8(13 \%)$ patients. Therefore, determination of the presence and number of tributary veins using all 6 images reconstructed throughout the cardiac cycle was necessary to identify the tributary veins.

In the 64 patients in whom the CVS could be evaluated, the best image was one of the Sys-Images in 39 patients (61\%; Fig 4A) and was one of the Dia-Images in 25 patients (39\%; Fig 4B).

\section{Detection and Number of Coronary Veins}

The CS and MCV were visible in all 64 patients in whom the CVS could be evaluated. The LMV and PV also were identified in $54(84 \%)$ and 60 (94\%) patients, respectively. On the lateral aspect of the LV between the AIV and the MCV, 2 veins were visualized in $27(42 \%)$ patients, 3 were visualized in $20(31 \%)$ patients, 4 were visualized in 7 (11\%) patients, 5 were visualized in $2(3 \%)$ patients and 1 was visualized in $8(13 \%)$ patients on the lateral aspect of the LV (Fig 5).

\section{Discussion}

\section{Major Findings}

Visual assessment of the CVS using MDCT had the following results: (1) evaluation of the CVS using MDCT was possible in most $(91 \%)$ of the patients; (2) the diameter of the CVS is greater in the Sys-Images than in the DiaImages, but motion artifact was observed more often in the Sys-Images; and (3) on the lateral aspect of the LV, at least 1 vein was invariably detected, and 2 veins were visualized in $36 \%$ of patients and more than 2 veins were visualized in $45 \%$ of patients.

These results indicate that MDCT can be used to noninvasively evaluate the CVS and that it is important to evaluate several images reconstructed from data acquired during both systole and diastole to identify the tributary veins.

\section{Other Noninvasive Modalities for Evaluation of the CVS}

Several noninvasive methods are used to detect and evaluate the CVS, with echocardiography being possibly the most usual! ${ }^{10,11}$ Although it may provide information on the $\mathrm{CS}$ or the proximal potion of the MCV, detailed assessment of the GCV, AIV, or the tributary veins of the CVS is difficult 10,11 and furthermore, successful imaging is influenced by the body condition of the patient and may also depend on the skill of the examiner.

Based on improved spatial, temporal, and contrast resolution, contrast enhanced electron-beam CT (EBCT) has become the preferred method for evaluating the CVS!2,13 However, Schaffler et al found that although the CS, MCV, and AIV could be detected in all patients, the detection rate for the PV, LMV, or small cardiac veins was low (13$38 \%) ! 2$ Gerber et al also evaluated the CVS using 3-D reconstructed tomographic images acquired by EBCT, but the quality of the 3-D images was quite low and detailed analysis of the CVS, including the lumen characteristics (eg, stenosis, branching) or the course of its tributary veins, was difficult! ${ }^{3}$

Newer MDCT scanners allow the rapid acquisition of images using thin collimation, thereby generating data sets that yield excellent 3-D and multiplanar renderings that have been shown to be accurate in depicting coronary artery stenosis ${ }^{14-19}$ In the present study, we demonstrated for the first time that MDCT could provide accurate and detailed information concerning the CVS and its tributary veins in most patients $(91 \%)$. Therefore, we believe that it 
is a useful modality for the assessment of the CVS.

\section{Diameter of the CVS During the Cardiac Cycle}

It is well known that coronary venous flow shows a phasic pattern during the cardiac cycle and that coronary sinus flow is usually predominant in diastole?22,23 Because the blood flow in the CVS has phasic variations during the cardiac cycle, optimal positioning of the image reconstruction window is crucial for achieving image quality. In the present study, the diameter of the CVS was greater in images reconstructed during systole than in those obtained during diastole, and therefore the detection of the origin of the tributary veins of the CVS was usually easier when the images reconstructed during systole were used. It may be that the evaluation of the CVS using reconstructed images only during diastole will underestimate the diameter of the CVS and the number of tributary veins. Therefore, it is important to evaluate the CVS using the systolic images when the number and diameter of tributary veins are to be examined. However, as mentioned earlier, there were usually fewer artifacts in the diastolic images, so we recommend using both.

\section{Clinical Implications}

The CVS is used increasingly for LV or biventricular pacing in patients with advanced heart failure and a wide QRS complex. LV pacing can be accomplished by percutaneous insertion of a pacing lead into a vein overlying the LV and the optimal site may be the mid-lateral wall of the $\mathrm{LV}$, corresponding to the location of the LMV or PV. However, technical limitations imposed by the CS and coronary venous anatomy result in a 10-15\% failure rate for LV lead placement and effective biventricular pacing! ${ }^{1-3}$ Hypoplasia of the sinus, narrowing or atresia of the ostium, and diverticulum are known anomalies, ${ }^{24,25}$ which MDCT can detect noninvasively, as well as the most suitable vein for LV or biventricular pacing, before the operation. Furthermore, even if the correct vein is present at the mid-lateral wall of the LV, cannulation of it or placing a pacing lead in the optimal position can be difficult depending on the angle to the GCV or CS and the diameter and course of the vein. Therefore, prior knowledge of the coronary venous anatomy from 3-D images may help in assessing the suitability of the vein for insertion of an LV pacemaker lead and in planning the procedural strategies. Thus, MDCT can pre-operatively provide useful information on the presence, course, branching angle from the CS and availability of coronary veins on the lateral aspect of the LV in most patients.

\section{Study Limitations}

First, because the diameter of the CVS was visually assessed using 3-D images, quantitative analysis could not be performed. To measure it precisely, multiplanner reconstruction images should have been used ${ }^{14-19}$ However, because of the presence of the phasic changes in the diameter of coronary veins during the cardiac cycle ${ }^{22,23}$ measurement of the actual diameter might be difficult. To determine the maximum diameter of the tributary veins and to assess their use for LV pacing, retrograde venography after occluding the CS lumen by a balloon may be necessary. Second, there was no pretreatment with $\beta$-blockers before MDCT. A patient's heart rate during image acquisition critically influences image quality 15 but although the efficacy of pretreatment with $\beta$-blockers before MDCT has been reported, ${ }^{16-19}$ it may be contraindicated in patients with advanced heart failure who are candidates for biventricular or LV pacing. Therefore, we did not administer $\beta$-blockers. Third, high-quality 3-D images cannot be obtained when there is frequent arrhythmia during image acquisition or there is a pacemaker lead or artificial valve. Therefore, at present, analysis of the CVS using 3-D images reconstructed by an 8-detector CT is only suitable for patients with normal sinus rhythm and no artificial materials near or in the heart. Finally, the sample size was relatively small, and the utility of MDCT was examined only in patients without structural heart disease. Furthermore, a 16-row MDCT scanner has recently been developed ${ }^{26}$ and therefore future studies including a larger number of patients with structural heart disease may be needed to further determine the usefulness of MDCT.

\section{Conclusions}

MDCT can evaluate the CVS and may provide useful information on the presence, number, and availability of coronary veins on the lateral aspect of the LV. It is important to evaluate several images reconstructed from the data acquired both during systole and diastole for precise identification.

\section{Acknowledgments}

The authors are indebted to the radiology technologists for their technical support. This work was supported by a Research Grant for Cardiovascular Diseases (14C-2) from the Ministry of Health, Labor and Welfare, Japan.

\section{References}

1. Gras D, Mabo P, Tang T, Luttikuis O, Chatoor R, Pedersen AK, et al. Multisite pacing as a supplemental treatment of congestive heart failure: Preliminary results of the Medtronic Inc. InSync Study. Pacing Clin Electrophysiol 1998; 21(Pt II): 2249-2255.

2. Daubert JC, Ritter P, Le Breton H, Gras D, Leclercq C, Lazarus A, et al. Permanent left ventricular pacing with transvenous leads inserted into the coronary veins. Pacing Clin Electrophysiol 1998; 21(Pt II): $239-245$.

3. Abraham WT, Fisher WG, Smith AL, Delurgio DB, Leon AR, Loh $\mathrm{E}$, et al. Cardiac resynchronization in chronic heart failure. $N$ Engl $J$ Med 2002; 346: 1845-1853.

4. Stellbrink C, Diem B, Schauerte P, Ziegert K, Hanrath P. Transcoronary venous radiofrequency catheter ablation of ventricular tachycardia. J Cardiovasc Electrophysiol 1997; 8: 916-921.

5. Cappato R, Schluter M, Weiss C, Willems S, Meinertz T, Kuck KH. Mapping of the coronary sinus and great cardiac vein using a 2French electrode catheter and a right femoral approach. J Cardiovasc Electrophysiol 1997; 8: 371-376.

6. Kenknight BH, Walker RG, Ideker RE. Marked reduction of ventricular defibrillation threshold by application of an auxiliary shock to a catheter electrode in the left posterior coronary vein of dogs. $J$ Cardiovasc Electrophysiol 2000; 11: 900-906.

7. Butter C, Meisel E, Engelmann L, Eldar M, Fleck E, Schubert B, et al. Human experience with transvenous biventricular defibrillation using an electrode in a left ventricular vein. Pacing Clin Electrophysiol 2002; 25: 324-331.

8. Meisel E, Pfeiffer D, Engelmann L, Tebbenjohanns J, Schubert B, Hahn S, et al. Investigation of coronary venous anatomy by retrograde venography in patients with malignant ventricular tachycardia. Circulation 2001; 104: 442-447.

9. Gilard M, Mansourati J, Etienne Y, Larlet JM, Truong B, Boschat J, et al. Angiographic anatomy of the coronary sinus and its tributaries. Pacing Clin Electrophysiol 1998; 21(Pt 2): 2280-2284.

10. D'Cruz IA, Shala MB, Johns C. Echocardiography of the coronary sinus in adults. Clin Cardiol 2000; 23: 149-154.

11. Omran H, Pfeiffer D, Tebbenjohanns J, Schumacher B, Manz M, Lauck G, et al. Echocardiographic imaging of coronary sinus diverticula and middle cardiac veins in patients with preexcitation syndrome: Impact on radiofrequency catheter ablation of posteroseptal 
accessory pathways. Pacing Clin Electrophysiol 1995; 18: 1236 1243.

12. Schaffler GJ, Groell R, Peichel KH, Rienmuller R. Imaging the coronary venous drainage system using electron-beam CT. Surg Radiol Anat 2000; 22: 35-39.

13. Gerber TC, Sheedy PF, Bell MR, Hayes DL, Rumberger JA, Behrenbeck T, et al. Evaluation of the coronary venous system using electron beam computed tomography. Int $J$ Cardiovasc Imaging 2001; 17: 65-75.

14. Becker CR, Ohnesorge BM, Schoepf UJ, Reiser MF. Current development of cardiac imaging with multidetector-row CT. Eur J Radiol 2003; 36: $97-103$.

15. Giesler T, Baum U, Ropers D, Ulzheimer S, Wenkel E, Mennicke M, et al. Noninvasive visualization of coronary arteries using contrastenhanced multidetector CT: Influence of heart rate on image quality and stenosis detection. AJR Am J Roemtgenol 2002; 179: 911-916.

16. Ropers D, Baum U, Pohle K, Anders K, Ulzheimer S, Ohnesorge B, et al. Detection of coronary artery stenoses with thin-slice multi-detector row spiral computed tomography and multiplanar reconstruction. Circulation 2003; 107: 664-666.

17. Sato Y, Matsumoto N, Kato M, Inoue F, Horie T, Kusama J, et al. Noninvasive assessment of coronary artery disease by multislice spiral computed tomography using a new retrospectively ECG-gated image reconstruction technique. Circ J 2003; 67: 401 - 405 .

18. Sato Y, Kato M, Inoue F, Fukui T, Imazeki T, Mitsui M, et al. Detection of coronary artery aneurysms, stenoses and occlusions by multislice spiral computed tomography in adolescents with Kawasaki disease. Circ J 2003; 67: 427-430.

19. Sato Y, Kanmatsuse K, Inoue F, Horie T, Kato M, Kusama J, et al. Noninvasive coronary artery imaging by multislice spiral computed tomography. Circ J 2003; 67: 107-111.

20. Scharf C, Sneider M, Case I, Chugh A, Lai SW, Pelosi F Jr, et al. Anatomy of the pulmonary veins in patients with atrial fibrillation and effects of segmental ostial ablation analyzed by computed tomography. J Cardiovasc Electrophysiol 2003; 14: 150-155.

21. Horiguchi J, Nakanishi T, Tamura A, Ito K, Sasaki K, Shen Y. Technical innovation of cardiac multirow detector CT using multisector reconstruction. Comput Med Imaging Graph 2002; 26: 217-226.

22. Canty JM, Brooks A. Phasic volumetric coronary venous outflow patterns in conscious dogs. Am J Physiol 1990; 258 (Heart Circ Physiol 27): H1457-H1463.

23. Chilian WM, Marcus ML. Coronary venous outflow persists after cessation of coronary arterial inflow. Am J Physiol 1984; 247(Pt 2): H984-H990.

24. Katritsis DG. The coronary sinus: Passive bystander or source of arrhythmia? HeartRhythm 2004; 1: 113-116.

25. Ho SY, Sanchez-Quintana D, Becker AE. A review of the coronary venous system: A road less traveled. HeartRhythm 2004; 1: 107112.

26. Mollet NR, Cademartiri F, Nieman K, Saia F, Lemos PA, McFadden $\mathrm{EP}$, et al. Multislice spiral computed tomography coronary angiography in patients with stable angina pectoris. J Am Coll Cardiol 2004; 43: $2265-2270$ 